\title{
Changes to virus taxonomy and the International Code of Virus Classification and Nomenclature ratified by the International Committee on Taxonomy of Viruses (2019)
}

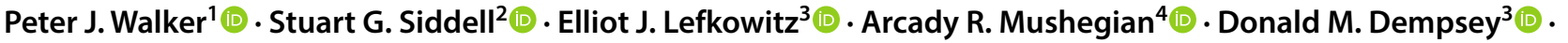

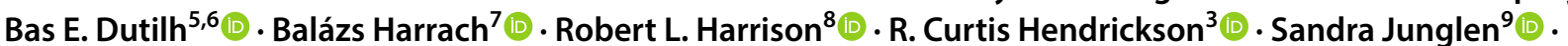

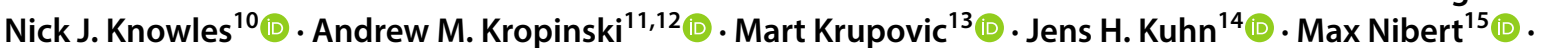

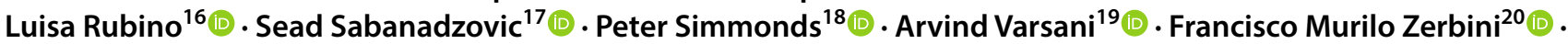 \\ Andrew J. Davison ${ }^{21}$ (i)
}

Published online: 12 June 2019

(c) This is a U.S. Government work and not under copyright protection in the US; foreign copyright protection may apply 2019

\begin{abstract}
This article reports the changes to virus taxonomy approved and ratified by the International Committee on Taxonomy of Viruses (ICTV) in February 2019. Of note, in addition to seven new virus families, the ICTV has approved, by an absolute majority, the creation of the realm Riboviria, a likely monophyletic group encompassing all viruses with positive-strand, negative-strand and double-strand genomic RNA that use cognate RNA-directed RNA polymerases for replication.
\end{abstract}

\section{Introduction}

Changes and updates to virus taxonomy (the universal scheme of virus classification of the International Committee on Taxonomy of Viruses [ICTV]) take place annually and are the result of a multi-stage process. In accordance with the ICTV Statutes (http://ictv.global/statutes.asp), proposals submitted to the ICTV Executive Committee (EC) undergo a review process that involves input from the ICTV Study Groups (SGs) and Subcommittees (SCs), other interested virologists, and the EC. After final approval by the $\mathrm{EC}$, proposals are presented for review and ratification to the full ICTV membership by publication on the ICTV website (http://ictv.global) followed by an electronic vote.

The latest set of proposals approved by the EC was made available on the ICTV website in January 2019 (see http:// ictv.global/proposals-2018/ for all proposals combined into a single zip file, and use the links provided in the References to access individual proposals). A list of proposals was then emailed on January 12, 2019 to the 162 members of ICTV, namely the EC Members, Life Members, ICTV

Handling Editor: Tim Skern

Arcady R. Mushegian

mushegian2@gmail.com

Extended author information available on the last page of the article
Subcommittee Members (including the SG chairs) and ICTV National Representatives. Members were then requested to vote on whether to ratify the taxonomic proposals. Voting closed on February 15, 2019.

\section{Changes to virus taxonomy, the International Code of Virus Classification and Nomenclature (ICTV Code), and the appointment of a new ICTV Life Member}

All proposals [1-216] were ratified by ICTV members, in every case receiving an absolute majority of votes, between 60 and $64 \%$ of eligible voters. A summary of the taxonomy changes enacted by the ratified proposals is provided in Table 1. Each proposal is cited and listed in the References to acknowledge the authors' efforts and to provide links to the specific document source on the ICTV website. These documents remain available for those who wish to see the full details.

The introduction, in late 2018, of additional ranks in virus taxonomy has necessitated several changes in the ICTV Code. The amended Code had been approved by the EC and has been ratified by ICTV voting on an additional ballot question. The Code can be found at http://ictv.global/ code.asp. 
Table 1 Summary of taxonomic changes approved in February 2019

\begin{tabular}{llllllll}
\hline Rank & Total, MSL-33 $^{\mathrm{a}}$ & New & Abolished & Renamed & Merged & Split & Total, MSL-34 $^{\mathrm{b}}$ \\
\hline Realm & 0 & 1 & 0 & 0 & 0 & 0 & 1 \\
Phylum & 1 & 0 & 0 & 0 & 0 & 0 & 1 \\
Subphylum & 2 & 0 & 0 & 0 & 0 & 0 & 2 \\
Class & 6 & 0 & 0 & 0 & 0 & 0 & 6 \\
Order & 14 & 0 & 0 & 0 & 0 & 0 & 14 \\
Suborder & 7 & 0 & 0 & 0 & 0 & 0 & 7 \\
Family & 143 & 7 & 0 & 0 & 0 & 0 & 150 \\
Subfamily & 64 & 15 & 0 & 0 & 0 & 0 & 79 \\
Genus & 846 & 175 & 0 & 137 & 0 & 2 & 1019 \\
Subgenus & 59 & 0 & 0 & 0 & 0 & 0 & 59 \\
Species & 4958 & 614 & 11 & 59 & 2 & 0 & 5,560 \\
\hline
\end{tabular}

${ }^{\text {a }}$ Total numbers of taxa in the ICTV Master Species List prior to 2019 ratification

$\mathrm{b}$ Total numbers of taxa now recognized, as reported in the ICTV Master Species List
Finally, Dr. Michael J. Adams has been elected as a Life Member, following nomination by the President, recommendation by the Executive Committee, and ratification by the ICTV membership [217]. Dr. Adams served the EC and ICTV in various capacities from 2001 till 2017, most recently as EC Business Secretary.

Perhaps the most notable taxonomic change approved in this ratification is the establishment of the realm Riboviria, a likely monophyletic clade of viruses with positivestrand, double-strand or negative-strand genomic RNA that use cognate RNA-directed RNA polymerases (RdRPs) for replication [2]. The realm Riboviria is placed at the highest taxonomic rank permitted by the ICTV Code. The evidence for monophyly of RNA viruses and for various clades within Riboviria has been accumulating over the years from phylogenetic analyses of the universal marker, RdRP, supplemented by comparison of additional molecular traits shared by subsets of RNA viruses [2, 218-220].

\section{Conclusion}

All proposals submitted to ratification were ratified by an absolute majority of the ICTV, and the changes proposed are now part of official ICTV taxonomy. An up-to-date list of all approved taxa can be found on the ICTV online website: http://ictv.global/msl.htm.

\section{Compliance with ethical standards}

Conflict of interest The authors declare no conflicts of interest. A.R.M. is a Program Director at the U.S. National Science Foundation (NSF); the statements and opinions expressed herein are made in a personal capacity and do not constitute endorsement by NSF or the government of the United States. Mention of trade names or commercial products in this publication is solely for the purpose of providing specific information and does not imply recommendation or endorsement by the U.S. Department of Agriculture. USDA is an Equal Opportunity Provider and Employer. The content of this publication does not necessarily reflect the views or policies of the U.S. Department of Health and Human Services or of the institutions and companies affiliated with the authors. This work was supported in part through Battelle Memorial Institute's prime contract with the U.S. National Institute of Allergy and Infectious Diseases (NIAID) under Contract No. HHSN272200700016I (J.H.K.). N.J.K. is partially supported by core funding provided by the Biotechnology and Biological Sciences Research Council, UK. B.E.D. is supported by Netherlands Organization for Scientific Research (NWO) Vidi Grant 864.14.004. B.H. is partially supported by National Research, Development and Innovation Office-NKFIH, NN128309. A.J.D. is supported by the Medical Research Council (MC_UU_12014/3). S.S. acknowledges partial support from Mississippi Agricultural and Forestry Experiment Station (MAFES), Mississippi State University.

Human/animal rights statement None of the work reported involved research on human participants or animals. All authors have contributed to this work and agreed to its publication. 
genus Betabaculovirus, family Baculoviridae 2018.002D. Robert.L.Harrison@ars.usda.gov. https://talk.ictvonline.org/ictv/ proposals/2018.002D.A.v1.Baculoviridae_8sp.zip. Accessed 17 Apr 2019

5. Varsani A (2018) Create ten new species in the genus Circovirus and two new species in the genus Cyclovirus, family Circoviridae 2018.003D. Arvind.varsani@asu.edu. https://talk.ictvonline.org/ ictv/proposals/2018.003D.A.v1.Circoviridae_12sp.zip. Accessed 17 Apr 2019

6. Chinchar VG, Yang F, Huang J, Williams T, Whittington R, Jancovich J, Subramaniamn K, Waltzek T, Hick P, Ince IA, Marschang R (2018) Create new genus Decapodiridovirus in the subfamily Betairidovirinae, family Iridoviridae 2018.004D. vchinchar@umc.edu. https://talk.ictvonline.org/ictv/propo sals/2018.004D.A.v1.Decapodiridovirus_1gen1sp.zip. Accessed 17 Apr 2019

7. Fehér E, Kaszab E, Lengyel G, Bányai K (2018) Create one new species in the genus Cyclovirus, family Circoviridae 2018.005D. feher.eniko@agrar.mta.hu. https://talk.ictvonline.org/ictv/propo sals/2018.005D.A.v1.Cyclovirus_sp.zip. Accessed 17 Apr 2019

8. Magnius L (2018) Create one new species in the genus Orthohepadnavirus, family Hepadnaviridae 2018.006D. Lars. magnius@gmail.com. https://talk.ictvonline.org/ictv/propo sals/2018.006D.A.v1.Orthohepadnavirus_sp.zip. Accessed 17 Apr 2019

9. Chinchar VG, Hick P, Jancovich J, Subramaniam K, Waltzek T, Whittington R, Williams T (2018) Create eight new species, remove three existing species in the family Iridoviridae 2018.007D.vchinchar@umc.edu. https://talk.ictvonline.org/ ictv/proposals/2018.007D.A.v1.Iridoviridae_8sp3sprem.zip. Accessed 17 Apr 2019

10. Calvignac-Spencer S, Carr M, Daugherty MD, Feltkamp MCW, Lauber C, Moens U, Verschoor EJ, Ehlers B (2018) Create ten new species and rename one species in the family Polyomaviridae 2018.008D. ehlersb@ rki.de. https://talk.ictvonline.org/ictv/ proposals/2018.008D.A.v1.Polyomaviridae_10sp.zip. Accessed 17 Apr 2019

11. Gatherer D, Benkő M, Brandt C, Bryant N, Dastjerdi A, Depledge D, Doszpoly A, Gompels U, Hartley C, Inoue N, Jarosinski K, Kaul R, Lacoste V, Norberg P, Origgi F, Orton R, Pellett P, Schmid S, Stewart J, Szpara M, Trimpert J, Vaz P, Waltzek T, Davison A, Beatty J, Jarvis M, Miller M, Troyer R (2018) Create eighteen new species in the family Herpesviridae, order Herpesvirales 2018.009D. andrew.davison@glasgow.ac.uk. https ://talk.ictvonline.org/ictv/proposals/2018.009D.A.v1.Herpesviri dae_18sp.zip. Accessed 17 Apr 2019

12. Penzes JJ (2018) Create one new species in the genus Dependoparvovirus, family Parvoviridae 2018.010D. Judit.penzes@ufl. edu. https://talk.ictvonline.org/ictv/proposals/2018.010D.A.v1. Dependoparvovirus_sp.zip. Accessed 17 Apr 2019

13. Khan AS, Kuhn JH, Johnson WE (2018) Create one new species, abolish one species, and correct one misspelled species name in the subfamily Spumaretrovirinae, family Retroviridae, order Ortervirales 2018.011D. welkin.johnson@bc.edu. https://talk. ictvonline.org/ictv/proposals/2018.011D.A.v1.Spumaretrovirin ae_sps.zip. Accessed 17 Apr 2019

14. Walker PJ, Blasdell KR, Dietzgen RG, Freitas-Astúa J, Kondo H, Tesh RB, Vasilakis N, Whitfield AE (2018) Create one new genus, Alphanemrhavirus, containing two new species in the family Rhabdoviridae, order Mononegavirales 2018.001M. peter.walker@uq.edu.au.https://talk.ictvonline.org/ictv/propo sals/2018.001M.A.v1.Alphanemrhavirus.zip. Accessed 17 Apr 2019

15. Walker PJ, Nylund A, Okland AL (2018) Create one new genus, Caligrhavirus, containing three new species in the family Rhabdoviridae, order Mononegavirales 2018.002M. peter.walker@uq.edu.au. https://talk.ictvonline.org/ictv/propo sals/2018.002M.A.v1.Caligrhavirus.zip. Accessed 17 Apr 2019

16. Freitas-Astúa J, Ramos-González PL, Chabi-Jesus C, Kitajima EW, Dietzgen RG (2018) Create three new species in the genus Dichorhavirus, family Rhabdoviridae, order Mononegavirales 2018.003M. juliana.astua@embrapa.br. https://talk.ictvonline .org/ictv/proposals/2018.003M.A.v1.Dichorhavirus_3sp.zip. Accessed 17 Apr 2019

17. Lelli D, Prosperi A, Moreno A, Chiapponi C, Sozzi E, Lavazza A (2018) Create one new species in the genus Ledantevirus, family Rhabdoviridae, order Mononegavirales 2018.004M. davide.lelli@izsler.it. https://talk.ictvonline.org/ictv/propo sals/2018.004M.A.v1.Ledantevirus_sp.zip. Accessed 17 Apr 2019

18. Buchmeier MJ, Charrel RN, Clegg CS, de la Torre JC, Gonzalez J-PJ, Günther S, Kuhn JH, Lukashevich IS, Radoshitzky SR, Romanowski V, Salvato MS, Sironi M, Stenglein MD (2018) Create one new genus, Antennavirus, containing two new species in the family Arenaviridae, order Bunyavirales 2018.005M. juanct@scripps.edu.https://talk.ictvonline.org/ictv/propo sals/2018.005M.A.v1.Antennavirus.zip Accessed 17 Apr 2019

19. Lambert AL, Adkins S, Alkhovsky S, Beer M, Blair C, Calisher CH, Drebot M, Hughes HR, Nunes MRT, Marklewitz M, Shi X-H (2018) Create thirty-eight new species in the genus Orthobunyavirus, family Peribunyaviridae, order Bunyavirales 2018.008M. 1tr8@cdc.gov. https://talk.ictvonline.org/ictv/propo sals/2018.008M.A.v3.Orthobunyavirus_38sp.zip. Accessed 17 Apr 2019

20. Ballinger MJ, Hall RA, Langevin SA, Pauvolid-Corrêa A, Junglen S (2018) Create five new species in the genus Orthophasmavirus, family Phasmaviridae, order Bunyavirales 2018.009M. Sandra.junglen@charite.de. https://talk.ictvonline.org/ictv/propo sals/2018.009M.A.v1.Phasmavirus_5sp.zip. Accessed 17 Apr 2019

21. Maes P, Calisher CH, Charbonnel N, Fulhorst CF, Klempa B, Klingström J, Song J-W, Zhang Y-Z (2018) Create four subfamilies and three new genera in the family Hantaviridae, order Bunyavirales 2018.010M. piet.maes@kuleuven.be. https://talk.ictvo nline.org/ictv/proposals/2018.010M.A.v3.Hantaviridae_4subf am.zip. Accessed 17 Apr 2019

22. Balkema-Buschmann A, Dundon WG, Duprex WP, Easton A.J, Fouchier RAM, Kurath G, Lamb RA, Lee B, Rima BK, Rota PA, Wang LF (2018) Re-organization of the family Paramyxoviridae, order Mononegavirales 2018.011M. b.rima@qub.ac.uk. https:// talk.ictvonline.org/ictv/proposals/2018.011M.A.v1.Paramyxovi ridae.zip. Accessed 17 Apr 2019

23. Kuhn JH (2018) Move one species (Estero Real orthobunyavirus) from the family Peribunyaviridae to the family Nairoviridae as species Estero Real orthonairovirus 2018.012M. kuhnjens@mail.nih.gov. https://talk.ictvonline.org/ictv/propo sals/2018.012M.A.v1.Bunyavirales_spmov.zip. Accessed 17 Apr 2019

24. Shen S, Duan X, Wang B, Zhu L, Zhang Y, Zhang J, Wang J, Luo T, Kou C, Liu D, Liu C, Zhang L, Chang C, Su Z, Tang S, Qiao J, Moming A, Wang C, Abudurexiti A, Wang H, Hu Z, Zhang Y, Sun S, Deng F (2018) Create one new species in the genus Banyangvirus, family Phenuiviridae, order Bunyavirales 2018.013M.df@wh.iov.cn. https://talk.ictvonline.org/ictv/propo sals/2018.013M.A.v1.Banyangvirus_sp.zip Accessed 17 Apr 2019

25. Matsuno K, Ebihara H (2018) Create one new species in the genus Phlebovirus, family Phenuiviridae, order Bunyavirales 2018.014M. Ebihara.Hideki@mayo.edu. https://talk.ictvo nline.org/ictv/proposals/2018.014M.A.v1.Phlebovirus_sp.zip. Accessed 17 Apr 2019 
26. Amarasinghe G, Basler C, Bavari S, Bukreyev AA, Chandran K, Crozier I, Dolnik O, Dye JM, Formenty PBH, Griffiths A, Hewson R, Kobinger G, Kuhn JH, Leroy EM, Mühlberger E, Netesov SV, Palacios G, Pályi B, Pawęska JT, Smither S, Takada A, Towner JS, Wahl V, Zhang T, Bao Y, Shi M, Holmes EC, Zhang Y (2018) Create two new genera, Striavirus and Thamnovirus, each containing one new species, in the family Filoviridae, order Mononegavirales 2018.015M. kuhnjens@mail.nih. gov. https://talk.ictvonline.org/ictv/proposals/2018.015M.A.v1. Filoviridae_2gen.zip. Accessed 17 Apr 2019

27. Rubbenstroth D, Briese T, Dürrwald R, Horie M, Hyndman T, Nowotny R, Payne S, Stenglein M, Tomonaga K, Kuhn JH (2018) Create one new genus, Cultervirus, containing one new species in the family Bornaviridae, order Mononegavirales 2018.016M. dennis.rubbenstroth@fli.de. https://talk.ictvonline.org/ictv/propo sals/2018.016M.A.v1.Cultervirus.zip Accessed 17 Apr 2019

28. Maes P, Kuhn JH (2018) Expansion of the order Bunyavirales 2018.017M. piet.maes@kuleuven.be.https://talk.ictvonline.org/ ictv/proposals/2018.017M.A.v4.Bunyavirales_2fam5gen.zip. Accessed 17 Apr 2019

29. Maes P, Kuhn JH (2018) Delete one species Wuhan fly phasivirus from the family Phenuiviridae, order Bunyavirales 2018.019M. kuhnjens@mail.nih.gov. https://talk.ictvonline.org/ictv/propo sals/2018.019M.A.v1.Phenuiviridae_Remsp.zip. Accessed 17 Apr 2019

30. Zell R, Gorbalenya AE, Hovi T, King AMQ, Knowles NJ, Lindberg AM, Oberste MS, Palmenberg AC, Reuter G, Simmonds P, Skern T, Tapparel C, Wolthers KC, Woo PCY (2018) Create one new genus, Ailurivirus, containing one species (Ailurivirus A) in the family Picornaviridae, order Picornavirales 2018.001S. roland.zell@med.uni-jena.de. https://talk.ictvonline.org/ictv/ proposals/2018.001S.A.v1.Ailurivirus.zip. Accessed 17 Apr 2019

31. Zell R, Gorbalenya AE, Hovi T, King AMQ, Knowles NJ, Lindberg AM, Oberste MS, Palmenberg AC, Reuter G, Simmonds P, Skern T, Tapparel C, Wolthers KC, Woo PCY (2018) Change the name of one species Avian sapelovirus (genus Sapelovirus) to Anativirus $A$ and move it to a new genus, Anativirus, in the family Picornaviridae, order Picornavirales 2018.002S. roland. zell@med.uni-jena.de. https://talk.ictvonline.org/ictv/propo sals/2018.002S.A.v1.Anativirus.zip. Accessed 17 Apr 2019

32. Zell R, Gorbalenya AE, Hovi T, King AMQ, Knowles NJ, Lindberg AM, Oberste MS, Palmenberg AC, Reuter G, Simmonds P, Skern T, Tapparel C, Wolthers KC, Woo PCY (2018) Create one new species (Cadicivirus B) in the genus Dicipivirus, family Picornaviridae, order Picornavirales 2018.003S. roland.zell@med.uni-jena.de https://talk.ictvonline.org/ictv/ proposals/2018.003S.A.v1.Dicipivirus_sp.zip. Accessed 17 Apr 2019

33. Zell R, Gorbalenya AE, Hovi T, King AMQ, Knowles NJ, Lindberg AM, Oberste MS, Palmenberg AC, Reuter G, Simmonds P, Skern T, Tapparel C, Wolthers KC, Woo PCY (2018) Create one new genus, Livupivirus, containing one species (Livupivirus A) in the family Picornaviridae, order Picornavirales 2018.004S. roland.zell@med.uni-jena.de. https://talk.ictvonline.org/ictv/ proposals/2018.004S.A.v1.Livupivirus.zip. Accessed 17 Apr 2019

34. Zell R, Gorbalenya AE, Hovi T, King AMQ, Knowles NJ, Lindberg AM, Oberste MS, Palmenberg AC, Reuter G, Simmonds P, Skern T, Tapparel C, Wolthers KC, Woo PCY (2018) Create one new genus, Malagasivirus, containing two species (Malagasivirus $A$ and Malagasivirus $B$ ) in the family Picornaviridae, order Picornavirales 2018.005S. roland.zell@med.uni-jena.de. https ://talk.ictvonline.org/ictv/proposals/2018.005S.A.v1.Malagasivi rus.zip. Accessed 17 Apr 2019
35. Zell R, Gorbalenya AE, Hovi T, King AMQ, Knowles NJ, Lindberg AM, Oberste MS, Palmenberg AC, Reuter G, Simmonds P, Skern T, Tapparel C, Wolthers KC, Woo PCY (2018) Create one new species (Mischivirus D) in the genus Mischivirus, family Picornaviridae, order Picornavirales 2018.006S. roland. zell@med.uni-jena.de. https://talk.ictvonline.org/ictv/propo sals/2018.006S.A.v1.Mischivirus_sp.zip. Accessed 17 Apr 2019

36. Zell R, Gorbalenya AE, Hovi T, King AMQ, Knowles NJ, Lindberg AM, Oberste MS, Palmenberg AC, Reuter G, Simmonds P, Skern T, Tapparel C, Wolthers KC, Woo PCY (2018) Create one new species (Passerivirus B) in the genus Passerivirus, family Picornaviridae, order Picornavirales 2018.007S. roland. zell@med.uni-jena.de. https://talk.ictvonline.org/ictv/propo sals/2018.007S.A.v1.Passerivirus_sp.zip. Accessed 17 Apr 2019

37. Zell R, Gorbalenya AE, Hovi T, King AMQ, Knowles NJ, Lindberg AM, Oberste MS, Palmenberg AC, Reuter G, Simmonds P, Skern T, Tapparel C, Wolthers KC, Woo PCY (2018) Create one new genus, Poecivirus, containing one species (Poecivirus A) in the family Picornaviridae, order Picornavirales 2018.008S. roland.zell@med.uni-jena.de. https://talk.ictvonline.org/ictv/ proposals/2018.008S.A.v1.Poecivirus.zip. Accessed 17 Apr 2019

38. Zell R, Gorbalenya AE, Hovi T, King AMQ, Knowles NJ, Lindberg AM, Oberste MS, Palmenberg AC, Reuter G, Simmonds P, Skern T, Tapparel C, Wolthers KC, Woo PCY (2018) Create three new species (Rabovirus B, Rabovirus $C$, Rabovirus $D$ ) in the genus Rabovirus, family Picornaviridae, order Picornavirales 2018.009S. roland.zell@ med.uni-jena.de. https://talk.ictvo nline.org/ictv/proposals/2018.009S.A.v1.Rabovirus_3sp.zip. Accessed 17 Apr 2019

39. Zell R, Gorbalenya AE, Hovi T, King AMQ, Knowles NJ, Lindberg AM, Oberste MS, Palmenberg AC, Reuter G, Simmonds P, Skern T, Tapparel C, Wolthers KC, Woo PCY (2018) Create one new genus, Rafivirus, containing two species (Rafivirus A, Rafivirus B) in the family Picornaviridae, order Picornavirales 2018.010S.roland.zell@med.uni-jena.de.https://talk.ictvonline .org/ictv/proposals/2018.010S.A.v1.Rafivirus.zip. Accessed 17 Apr 2019

40. Zell R, Gorbalenya AE, Hovi T, King AMQ, Knowles NJ, Lindberg AM, Oberste MS, Palmenberg AC, Reuter G, Simmonds P, Skern T, Tapparel C, Wolthers KC, Woo PCY (2018) Create two new species (Rosavirus B, Rosavirus C) in the genus Rosavirus, family Picornaviridae, order Picornavirales 2018.011S. roland. zell@med.uni-jena.de. https://talk.ictvonline.org/ictv/propo sals/2018.011S.A.v1.Rosavirus_2sp.zip. Accessed 17 Apr 2019

41. Zell R, Gorbalenya AE, Hovi T, King AMQ, Knowles NJ, Lindberg AM, Oberste MS, Palmenberg AC, Reuter G, Simmonds P, Skern T, Tapparel C, Wolthers KC, Woo PCY (2018) Create one new genus, Tottorivirus, containing one species (Tottorivirus A) in the family Picornaviridae, order Picornavirales 2018.012S. roland.zell@med.uni-jena.de. https://talk.ictvonline.org/ictv/ proposals/2018.012S.A.v1.Tottorivirus.zip. Accessed 17 Apr 2019

42. Chen R-B, Mukhopadhyay S, Merits A, Bolling B, Nasar F, Coffey LL, Powers A, Weaver SC, Smith D, Simmonds P, Siddell S (2018) Create one new unassigned family Matonaviridae to contain the genus Rubivirus, moved from the family Togaviridae 2018.013S. rubing.chen@gmail.com. https://talk.ictvonline.org/ ictv/proposals/2018.013S.A.v3.Matonaviridae.zip. Accessed 17 Apr 2019

43. Vinjé J, Esteves P, Estes M, Green K, Katayama K, Knowles NJ, L'Homme Y, Martella V, Vennema H, White P (2018) Create six genera (Bavovirus, Minovirus, Nacovirus, Recovirus, Salvovirus, Valovirus) in the family Caliciviridae 2018.014S. jvinje@cdc. gov. https://talk.ictvonline.org/ictv/proposals/2018.014S.A.v2. Caliciviridae_6gen.zip. Accessed 17 Apr 2019 
44. Kropinski AM, Adriaenssens E (2018) Create one new genus, Iodovirus, containing one new species, in the family Myoviridae, order Caudovirales 2018.001B. Phage.Canada@gmail.com. https ://talk.ictvonline.org/ictv/proposals/2018.001B.A.v1.Iodovirus. zip. Accessed 17 Apr 2019

45. Kropinski AM, Kuhn JH, Adriaenssens EM (2018) Correct the misspelled name of species Gordonia virus Nymphadora in the genus Nymphadoravirus, family Siphoviridae, order Caudovirales 2018.002B. Phage.Canada@gmail.com. https://talk.ictvo nline.org/ictv/proposals/2018.002B.A.v1.Nymphadoravirus.ren. zip. Accessed 17 Apr 2019

46. Kropinski AM, Adriaenssens EM, Zamani I, Bouzari M, Emtiazi G, Ghasemi SM, Esfahani SA, Chang H-I, Yazdi M (2018) Create one new genus, Farahnazvirus, containing one new species, in the family Siphoviridae, order Caudovirales 2018.003B. Phage.Canada@gmail.com. https://talk.ictvonline.org/ictv/propo sals/2018.003B.A.v1.Farahnazvirus.zip. Accessed 17 Apr 2019

47. Ghasemi SM, Esfahani SA, Bouzari M, Chang H-I, Yazdi M (2018) Create one new genus, Negarvirus, containing one new species, in the subfamily Picovirinae, family Podoviridae, order Caudovirales 2018.004B. bouzari@sci.ui.ac.ir. https://talk. ictvonline.org/ictv/proposals/2018.004B.A.v3.Negarvirus.zip. Accessed 17 Apr 2019

48. Kropinski AM, Adriaenssens EM (2018) Create one new genus, Iapetusvirus, containing one new species, in the family Myoviridae, order Caudovirales 2018.005B. Phage.Canada@gmail.com. https://talk.ictvonline.org/ictv/proposals/2018.005B.A.v1.Iapet usvirus.zip. Accessed 17 Apr 2019

49. Kropinski AM, Adriaenssens EM (2018) Create one new genus, Noxifervirus, containing one new species, in the family Myoviridae, order Caudovirales 2018.006B. Phage.Canada@gmail.com. https://talk.ictvonline.org/ictv/proposals/2018.006B.A.v1.Noxif ervirus.zip. Accessed 17 Apr 2019

50. Uchiyama J, Jang H-B, Klumpp J, Petty NK, Prangishvili D, Aziz RK, Brister RJ, van Zyl L, Edwards R, Oksanen H, Tong Y, Wittmann J, Kropinski AM, Rumnieks J, Clokie M, Dutilh BE, Alfenas-Zerbinin P, Duffy S, Lavigne R, Gillis A, Enault F, Lobocka M, Adriaenssens E, Sullivan M, Knezevic P, Barylsk J, Poranen M, Turner D, Krupovic M, Switt AM (2018) Rename 137 phage genera and six species in the order Caudovirales 2018.007B. Phage.Canada@gmail.com. https://talk.ictvo nline.org/ictv/proposals/2018.007B.A.v4.rename137gen6sp.zip. Accessed 17 Apr 2019

51. Kropinski AM, Adriaenssens EM (2018) Create one new genus, Bantamvirus, containing one new species, in the family Siphoviridae, order Caudovirales 2018.008B. Phage. Canada@gmail.com. https://talk.ictvonline.org/ictv/propo sals/2018.008B.A.v1.Bantamvirus.zip. Accessed 17 Apr 2019

52. Kropinski AM, Adriaenssens EM (2018) Create one new genus, Bendigovirus, containing one new species, in the family Siphoviridae, order Caudovirales 2018.009B. Phage. Canada@gmail.com. https://talk.ictvonline.org/ictv/propo sals/2018.009B.A.v2.Bendigovirus.zip. Accessed 17 Apr 2019

53. Kropinski AM, Adriaenssens EM (2018) Create one new genus, Eyrevirus, containing one new species, in the family Siphoviridae, order Caudovirales 2018.010B. Phage. Canada@gmail.com. https://talk.ictvonline.org/ictv/propo sals/2018.010B.A.v1.Eyrevirus.zip. Accessed 17 Apr 2019

54. Kropinski AM, Adriaenssens EM (2018) Create one new genus, Galunavirus, containing one new species, in the family Siphoviridae, order Caudovirales 2018.011B. Phage. Canada@gmail.com. https://talk.ictvonline.org/ictv/propo sals/2018.011B.A.v1.Galunavirus.zip. Accessed 17 Apr 2019

55. Kropinski AM, Adriaenssens EM (2018) Create one new genus, Gamtrevirus, containing one new species, in the family Siphoviridae, order Caudovirales 2018.012B. Phage.
Canada@gmail.com. https://talk.ictvonline.org/ictv/propo sals/2018.012B.A.v1.Gamtrevirus.zip. Accessed 17 Apr 2019

56. Kropinski AM, Adriaenssens EM (2018) Create one new genus, Gorjumvirus, containing one new species, in the family Siphoviridae, order Caudovirales 2018.013B. Phage. Canada@gmail.com. https://talk.ictvonline.org/ictv/propo sals/2018.013B.A.v1.Gorjumvirus.zip. Accessed 17 Apr 2019

57. Kropinski AM, Adriaenssens EM (2018) Create one new genus, Jasminevirus, containing two new species, in the family Podoviridae, order Caudovirales 2018.014B. Phage. Canada@gmail.com. https://talk.ictvonline.org/ictv/propo sals/2018.014B.A.v1.Jasminevirus.zip. Accessed 17 Apr 2019

58. Kropinski AM, Adriaenssens EM (2018) Create one new genus, Orchidvirus, containing one new species, in the family Siphoviridae, order Caudovirales 2018.016B. Phage. Canada@gmail.com. https://talk.ictvonline.org/ictv/propo sals/2018.016B.A.v1.Orchidvirus.zip. Accessed 17 Apr 2019

59. Kropinski AM, Adriaenssens EM (2018) Create one new genus, Poushouvirus, containing one new species, in the family Siphoviridae, order Caudovirales 2018.017B. Phage. Canada@gmail.com. https://talk.ictvonline.org/ictv/propo sals/2018.017B.A.v1.Poushouvirus.zip. Accessed 17 Apr 2019

60. Kropinski AM, Adriaenssens EM (2018) Create one new genus, Yvonnevirus, containing one new species, in the family Siphoviridae, order Caudovirales 2018.018B. Phage. Canada@gmail.com. https://talk.ictvonline.org/ictv/propo sals/2018.018B.A.v1.Yvonnevirus.zip. Accessed 17 Apr 2019

61. Kropinski AM, Adriaenssens EM (2018) Create one new genus, Bowservirus, containing one new species, in the family Siphoviridae, order Caudovirales 2018.019B. Phage. Canada@gmail.com. https://talk.ictvonline.org/ictv/propo sals/2018.019B.A.v1.Bowservirus.zip. Accessed 17 Apr 2019

62. Kropinski AM, Adriaenssens EM (2018) Create one new genus, Galaxyvirus, containing two new species, in the family Siphoviridae, order Caudovirales 2018.020B. Phage. Canada@gmail.com. https://talk.ictvonline.org/ictv/propo sals/2018.020B.A.v1.Galaxyvirus.zip. Accessed 17 Apr 2019

63. Kropinski AM, Adriaenssens EM (2018) Create one new genus, Gesputvirus, containing one new species, in the family Siphoviridae, order Caudovirales 2018.021B. Phage. Canada@gmail.com. https://talk.ictvonline.org/ictv/propo sals/2018.021B.A.v1.Gesputvirus.zip. Accessed 17 Apr 2019

64. Kropinski AM, Adriaenssens EM (2018) Create one new genus, Ghobesvirus, containing one new species, in the family Siphoviridae, order Caudovirales 2018.022B.Phage.Canada@gmail. com. https://talk.ictvonline.org/ictv/proposals/2018.022B.A.v1. Ghobesvirus.zip. Accessed 17 Apr 2019

65. Kropinski AM, Adriaenssens EM (2018) Create one new genus, Gustavvirus, containing two new species, in the family Siphoviridae, order Caudovirales 2018.023B.Phage.Canada@gmail. com. https://talk.ictvonline.org/ictv/proposals/2018.023B.A.v1. Gustavvirus.zip. Accessed 17 Apr 2019

66. Kropinski AM, Adriaenssens EM (2018) Create one new genus, Alcyoneusvirus, containing two new species, in the family Myoviridae, order Caudovirales 2018.024B. Phage.Canada@gmail. com. https://talk.ictvonline.org/ictv/proposals/2018.024B.A.v2. Alcyoneusvirus.zip. Accessed 17 Apr 2019

67. Kropinski AM, Adriaenssens EM (2018) Create one new genus, Asteriusvirus, containing two new species, in the family Myoviridae, order Caudovirales 2018.025B. Phage.Canada@gmail.com. https://talk.ictvonline.org/ictv/proposals/2018.025B.A.v1.Aster iusvirus.zip. Accessed 17 Apr 2019

68. Kropinski AM, Adriaenssens EM (2018) Create one new genus, Eneladusvirus, containing two species, in the family Myoviridae, order Caudovirales 2018.026B. Phage.Canada@gmail.com. https 
://talk.ictvonline.org/ictv/proposals/2018.026B.A.v1.Eneladusvi rus.zip. Accessed 17 Apr 2019

69. Kropinski AM, Adriaenssens EM (2018) Create one new genus, Mimasvirus, containing two new species, in the family Myoviridae, order Caudovirales 2018.027B. Phage.Canada@gmail.com. https://talk.ictvonline.org/ictv/proposals/2018.027B.A.v1.Mimas virus.zip. Accessed 17 Apr 2019

70. Kropinski AM, Adriaenssens EM (2018) Create one new genus, Hedwigvirus, containing one new species, in the family Siphoviridae, order Caudovirales 2018.028B.Phage.Canada@gmail. com. https://talk.ictvonline.org/ictv/proposals/2018.028B.A.v1. Hedwigvirus.zip. Accessed 17 Apr 2019

71. Kropinski AM, Adriaenssens EM (2018) Create one new genus, Nyceiraevirus, containing one new species, in the family Siphoviridae, order Caudovirales 2018.029B. Phage.Canada@gmail. com. https://talk.ictvonline.org/ictv/proposals/2018.029B.A.v1. Nyceiraevirus.zip. Accessed 17 Apr 2019

72. Kropinski AM, Adriaenssens EM (2018) Create one new genus, Polybotosvirus, containing one new species, in the family Myoviridae, order Caudovirales 2018.030B. Phage.Canada@gmail. com. https://talk.ictvonline.org/ictv/proposals/2018.030B.A.v1. Polybotosvirus.zip. Accessed 17 Apr 2019

73. Kropinski AM, Adriaenssens EM (2018) Create one new genus, Tortellinivirus, containing one new species, in the family Siphoviridae, order Caudovirales 2018.031B. Phage.Canada@gmail. com. https://talk.ictvonline.org/ictv/proposals/2018.031B.A.v1. Tortellinivirus.zip. Accessed 17 Apr 2019

74. Kropinski AM, Adriaenssens EM (2018) Create one new genus, Bingvirus, containing one new species, in the family Siphoviridae, order Caudovirales 2018.032B. Phage.Canada@gmail.com. https://talk.ictvonline.org/ictv/proposals/2018.032B.A.v1.Bingv irus.zip. Accessed 17 Apr 2019

75. Kropinski AM, Adriaenssens EM, Wittmann J (2018) Create one new genus, Cimpunavirus, containing one new species, in the family Siphoviridae, order Caudovirales 2018.033B. jow12@dsmz.de. https://talk.ictvonline.org/ictv/propo sals/2018.033B.A.v1.Cimpunavirus.zip. Accessed 17 Apr 2019

76. Kropinski AM, Adriaenssens EM (2018) Create one new genus, Cinunavirus, containing one new species, in the family Siphoviridae, order Caudovirales 2018.034B. Phage.Canada@gmail. com. https://talk.ictvonline.org/ictv/proposals/2018.034B.A.v1. Cinunavirus.zip. Accessed 17 Apr 2019

77. Kropinski AM, Adriaenssens EM (2018) Create one new genus, Dismasvirus, containing one new species, in the family Siphoviridae, order Caudovirales 2018.035B.Phage.Canada@gmail. com. https://talk.ictvonline.org/ictv/proposals/2018.035B.A.v1. Dismasvirus.zip. Accessed 17 Apr 2019

78. Kropinski AM, Adriaenssens EM (2018) Create one new genus, Elerivirus, containing one new species, in the family Siphoviridae, order Caudovirales 2018.036B. Phage.Canada@gmail.com. https://talk.ictvonline.org/ictv/proposals/2018.036B.A.v1.Eleri virus.zip. Accessed 17 Apr 2019

79. Kropinski AM, Adriaenssens EM (2018) Create one new genus, Ilzatvirus, containing two new species, in the family Siphoviridae, order Caudovirales 2018.037B. Phage.Canada@gmail.com. https://talk.ictvonline.org/ictv/proposals/2018.037B.A.v1.Ilzat virus.zip. Accessed 17 Apr 2019

80. Kropinski AM, Adriaenssens EM (2018) Create one new genus, Kojivirus, containing two new species, in the family Siphoviridae, order Caudovirales 2018.038B. Phage.Canada@gmail.com. https://talk.ictvonline.org/ictv/proposals/2018.038B.A.v1.Kojiv irus.zip. Accessed 17 Apr 2019

81. Kropinski AM, Adriaenssens EM (2018) Create one new genus, Minunavirus, containing one new species, in the family Siphoviridae, order Caudovirales 2018.039B. Phage.Canada@gmail. com. https://talk.ictvonline.org/ictv/proposals/2018.039B.A.v1. Minunavirus.zip. Accessed 17 Apr 2019

82. Kropinski AM, Adriaenssens EM (2018) Create one new genus, Pikminvirus, containing one new species, in the family Siphoviridae, order Caudovirales 2018.040B.Phage.Canada@gmail. com. https://talk.ictvonline.org/ictv/proposals/2018.040B.A.v1. Pikminvirus.zip. Accessed 17 Apr 2019

83. Kropinski AM, Adriaenssens EM (2018) Create one new genus, Rimavirus, containing two new species, in the family Siphoviridae, order Caudovirales 2018.041B. Phage.Canada@gmail.com. https://talk.ictvonline.org/ictv/proposals/2018.041B.A.v1.Rimav irus.zip. Accessed 17 Apr 2019

84. Kropinski AM, Adriaenssens EM (2018) Create one new genus, Scapunavirus, containing one new species, in the family Siphoviridae, order Caudovirales 2018.042B.Phage.Canada@gmail. com. https://talk.ictvonline.org/ictv/proposals/2018.042B.A.v1. Scapunavirus.zip. Accessed 17 Apr 2019

85. Kropinski AM, Adriaenssens EM (2018) Create one new genus, Vidquintavirus, containing one species, in the family Siphoviridae, order Caudovirales 2018.043B. Phage.Canada@gmail.com. https://talk.ictvonline.org/ictv/proposals/2018.043B.A.v1.Vidqu intavirus.zip. Accessed 17 Apr 2019

86. Kropinski AM, Adriaenssens EM (2018) Create one new species in the genus Amigovirus, family Siphoviridae, order Caudovirales 2018.044B. Phage.Canada@gmail.com. https://talk.ictvo nline.org/ictv/proposals/2018.044B.A.v1.Amigovirus_sp.zip. Accessed 17 Apr 2019

87. Kropinski AM, Adriaenssens EM (2018) Create one new genus, Ahduovirus, containing one new species, in the family Siphoviridae, order Caudovirales 2018.045B. Phage.Canada@gmail.com. https://talk.ictvonline.org/ictv/proposals/2018.045B.A.v1.Ahduo virus.zip. Accessed 17 Apr 2019

88. Kropinski AM, Adriaenssens EM, Krupovic M (2018) Create one new genus, Aqualcavirus, containing one new species, in the family Podoviridae, order Caudovirales 2018.046B. Phage. Canada@gmail.com. https://talk.ictvonline.org/ictv/propo sals/2018.046B.A.v1.Aqualcavirus.zip. Accessed 17 Apr 2019

89. Kropinski AM, Adriaenssens EM (2018) Create one new genus, Ceetrepovirus, containing three new species, in the family Siphoviridae, order Caudovirales 2018.047B. Phage.Canada@gmail. com. https://talk.ictvonline.org/ictv/proposals/2018.047B.A.v1. Ceetrepovirus.zip. Accessed 17 Apr 2019

90. Kropinski AM, Adriaenssens EM (2018) Create one new genus, Chunghsingvirus, containing one new species, in the family Siphoviridae, order Caudovirales 2018.048B. Phage. Canada@gmail.com. https://talk.ictvonline.org/ictv/propo sals/2018.048B.A.v1.Chunghsingvirus.zip. Accessed 17 Apr 2019

91. Kropinski AM, Adriaenssens EM (2018) Create one new genus, Gofduovirus, containing one new species, in the family Myoviridae, order Caudovirales 2018.049B. Phage.Canada@gmail.com. https://talk.ictvonline.org/ictv/proposals/2018.049B.A.v1.Gofdu ovirus.zip. Accessed 17 Apr 2019

92. Kropinski AM, Adriaenssens EM (2018) Create one new genus, Ikedavirus, containing two new species, in the family Siphoviridae, order Caudovirales 2018.050B. Phage.Canada@gmail.com. https://talk.ictvonline.org/ictv/proposals/2018.050B.A.v1.Ikeda virus.zip. Accessed 17 Apr 2019

93. Kropinski AM, Adriaenssens EM (2018) Create one new genus, Lilyvirus, containing one new species, in the family Siphoviridae, order Caudovirales 2018.052B. Phage.Canada@gmail.com. https ://talk.ictvonline.org/ictv/proposals/2018.052B.A.v1.Lilyvirus. zip. Accessed 17 Apr 2019

94. Kropinski AM, Adriaenssens EM (2018) Create one new genus, Nazgulvirus, containing one new species, in the 
family Myoviridae, order Caudovirales 2018.053B. Phage. Canada@gmail.com. https://talk.ictvonline.org/ictv/propo sals/2018.053B.A.v1.Nazgulvirus.zip. Accessed 17 Apr 2019

95. Kropinski AM, Adriaenssens EM (2018) Create one new genus, Popoffvirus, containing one new species, in the family Myoviridae, order Caudovirales 2018.054B. Phage.Canada@gmail.com. https://talk.ictvonline.org/ictv/proposals/2018.054B.A.v1.Popof fvirus.zip. Accessed 17 Apr 2019

96. Kropinski AM, Adriaenssens EM (2018) Create one new genus, Samistivirus, containing six new species, in the family Siphoviridae, order Caudovirales 2018.055B. Phage.Canada@gmail.com. https://talk.ictvonline.org/ictv/proposals/2018.055B.A.v1.Samis tivirus.zip. Accessed 17 Apr 2019

97. Kropinski AM, Adriaenssens EM, Petrovski S (2018) Create one new genus, Tijeunavirus, containing one new species, in the family Myoviridae, order Caudovirales 2018.056B. Phage. Canada@gmail.com. https://talk.ictvonline.org/ictv/propo sals/2018.056B.A.v1.Tijeunavirus.zip. Accessed 17 Apr 2019

98. Kropinski AM, Adriaenssens EM (2018) Create one new genus, Trinavirus, containing one new species, in the family Siphoviridae, order Caudovirales 2018.057B. Phage.Canada@gmail.com. https://talk.ictvonline.org/ictv/proposals/2018.057B.A.v1.Trina virus.zip. Accessed 17 Apr 2019

99. Kropinski AM, Adriaenssens EM (2018) Create one new genus, Vividuovirus, containing three new species, in the family Siphoviridae, order Caudovirales 2018.058B.Phage.Canada@gmail. com. https://talk.ictvonline.org/ictv/proposals/2018.058B.A.v1. Vividuovirus.zip. Accessed 17 Apr 2019

100. Kropinski AM, Adriaenssens EM (2018) Create one new genus, Weaselvirus, containing one new species, in the family Siphoviridae, order Caudovirales 2018.059B. Phage.Canada@gmail. com. https://talk.ictvonline.org/ictv/proposals/2018.059B.A.v1. Weaselvirus.zip. Accessed 17 Apr 2019

101. Kropinski AM, Adriaenssens EM, Krupovic M (2018) Create one new genus, Wilnyevirus, containing one new species, in the family Siphoviridae, order Caudovirales 2018.060B. Phage. Canada@gmail.com. https://talk.ictvonline.org/ictv/propo sals/2018.060B.A.v1.Wilnyevirus.zip. Accessed 17 Apr 2019

102. Kropinski AM, Adriaenssens EM (2018) Create one new genus, Aphroditevirus, containing one new species, in the family Myoviridae, order Caudovirales 2018.061B. Phage.Canada@gmail. com. https://talk.ictvonline.org/ictv/proposals/2018.061B.A.v1. Aphroditevirus.zip. Accessed 17 Apr 2019

103. Kropinski AM, Adriaenssens EM (2018) Create one new genus, Betterkatzvirus, containing one new species, in the family Siphoviridae, order Caudovirales 2018.062B. Phage.Canada@gmail. com. https://talk.ictvonline.org/ictv/proposals/2018.062B.A.v1. Betterkatzvirus.zip. Accessed 17 Apr 2019

104. Kropinski AM, Adriaenssens EM, Lavigne R, Turner D (2018) Create one new genus, Bifseptvirus, containing two new species, in the family Podoviridae, order Caudovirales 2018.063B. Phage.Canada@gmail.com. https://talk.ictvonline.org/ictv/propo sals/2018.063B.A.v1.Bifseptvirus.zip. Accessed 17 Apr 2019

105. Kropinski AM, Adriaenssens EM, Colberg O, Carstens AB, Kot W, Hansen LH (2018) Create one new genus, Bjornvirus, containing one new species, in the family Podoviridae, order Caudovirales 2018.064B. Phage.Canada@gmail.com. https:// talk.ictvonline.org/ictv/proposals/2018.064B.A.v1.Bjornvirus .zip. Accessed 17 Apr 2019

106. Kropinski AM, Adriaenssens EM (2018) Create one new genus, Britbratvirus, containing one new species, in the family Siphoviridae, order Caudovirales 2018.065B.Phage.Canada@gmail. com. https://talk.ictvonline.org/ictv/proposals/2018.065B.A.v1. Britbratvirus.zip. Accessed 17 Apr 2019

107. Kropinski AM, Adriaenssens EM (2018) Create one new genus, Brunovirus, containing one new species, in the family Myoviridae, order Caudovirales 2018.066B. Phage. Canada@gmail.com. https://talk.ictvonline.org/ictv/propo sals/2018.066B.A.v1.Brunovirus.zip. Accessed 17 Apr 2019

108. Kropinski AM, Adriaenssens EM, Bouzari M (2018) Create one new genus, Busanvirus, containing one new species, in the family Myoviridae, order Caudovirales 2018.067B. Phage. Canada@gmail.com. https://talk.ictvonline.org/ictv/propo sals/2018.067B.A.v1.Busanvirus.zip. Accessed 17 Apr 2019

109. Magill D, Kropinski AM, Adriaenssens EM, Kulakov L (2018) Create one new genus, Chakrabartyvirus, containing one new species, in the family Myoviridae, order Caudovirales 2018.068B. Phage.Canada@gmail.com. https://talk.ictvonline .org/ictv/proposals/2018.068B.A.v1.Chakrabartyvirus.zip. Accessed 17 Apr 2019

110. Kropinski AM, Adriaenssens EM, Jang B-H (2018) Create one new genus, Chungbukvirus, containing one new species, in the family Siphoviridae, order Caudovirales 2018.069B. Phage. Canada@gmail.com. https://talk.ictvonline.org/ictv/propo sals/2018.069B.A.v1.Chungbukvirus.zip. Accessed 17 Apr 2019

111. Kropinski AM, Adriaenssens EM (2018) Create one new genus, Emalynvirus, containing four new species, in the family Siphoviridae, order Caudovirales 2018.070B. Phage.Canada@gmail. com. https://talk.ictvonline.org/ictv/proposals/2018.070B.A.v1. Emalynvirus.zip. Accessed 17 Apr 2019

112. Kropinski AM, Adriaenssens EM, Klumpp J, Lavigne R (2018) Create one new genus, Flaumdravirus, containing two new species, in the family Myoviridae, order Caudovirales 2018.071B. Phage.Canada@gmail.com. https://talk.ictvonline.org/ictv/propo sals/2018.071B.A.v3.Flaumdravirus.zip. Accessed 17 Apr 2019

113. Kropinski AM, Adriaenssens EM (2018) Create one new genus, Getseptimavirus, containing two new species, in the family Siphoviridae, order Caudovirales 2018.072B. Phage. Canada@gmail.com. https://talk.ictvonline.org/ictv/propo sals/2018.072B.A.v1.Getseptimavirus.zip. Accessed 17 Apr 2019

114. Kropinski AM, Adriaenssens EM, Villamor J, Santos F, Antón J (2018) Create one new genus, Holosalinivirus, containing two new species, in the family Siphoviridae, order Caudovirales 2018.073B. Phage.Canada@gmail.com. https://talk.ictvo nline.org/ictv/proposals/2018.073B.A.v1.Holosalinivirus.zip. Accessed 17 Apr 2019

115. Kropinski AM, Ackermann H-W, Adriaenssens E (2018) Create one new genus, Ionavirus, containing one new species, in the family Myoviridae, order Caudovirales 2018.074B. Phage. Canada@gmail.com. https://talk.ictvonline.org/ictv/propo sals/2018.074B.A.v1.Ionavirus.zip. Accessed 17 Apr 2019

116. Kropinski AM, Adriaenssens EM (2018) Create one new genus, Sasvirus, containing one new species, in the family Siphoviridae, order Caudovirales 2018.075B. Phage. Canada@gmail.com. https://talk.ictvonline.org/ictv/propo sals/2018.075B.A.v1.Sasvirus.zip. Accessed 17 Apr 2019

117. Kropinski AM, Adriaenssens EM (2018) Create one new genus, Jedunavirus, containing three new species, in the family Myoviridae, order Caudovirales 2018.076B. Phage. Canada@gmail.com. https://talk.ictvonline.org/ictv/propo sals/2018.076B.A.v1.Jedunavirus.zip. Accessed 17 Apr 2019

118. Kropinski AM, Adriaenssens EM, Villamor J, Santos F, Antón J (2018) Create one new genus, Kairosalinivirus, containing two species, in the family Siphoviridae, order Caudovirales 2018.077B. Phage.Canada@gmail.com. https://talk.ictvonline .org/ictv/proposals/2018.077B.A.v1.Kairosalinivirus.zip. Accessed 17 Apr 2019

119. Kropinski AM, Adriaenssens EM, Kulakov L (2018) Create one new genus, Krylovvirus, containing one new species, in the family Podoviridae, order Caudovirales 2018.078B. Phage. 
Canada@gmail.com. https://talk.ictvonline.org/ictv/propo sals/2018.078B.A.v1.Krylovvirus.zip. Accessed 17 Apr 2019

120. Kropinski AM, Adriaenssens EM, Villamor J, Santos F, Antón J (2018) Create one new genus, Kryposalinivirus, containing two new species, in the family Siphoviridae, order Caudovirales 2018.079B. Phage.Canada@gmail.com. https://talk. ictvonline.org/ictv/proposals/2018.079B.A.v1.Kryptosalinivir us.zip. Accessed 17 Apr 2019

121. Kropinski AM, Adriaenssens EM, Nielsen TK, Carstens AB, Browne P, Neve H, Kot W, Hansen LH (2018) Create one new genus, Lacusarxvirus, containing one new species, in the family Siphoviridae, order Caudovirales 2018.080B. Phage. Canada@gmail.com. https://talk.ictvonline.org/ictv/propo sals/2018.080B.A.v1.Lacusarxvirus.zip. Accessed 17 Apr 2019

122. Kropinski AM, Adriaenssens EM (2018) Create one new genus, Myxoctovirus, containing one new species, in the family Podoviridae, order Caudovirales 2018.081B.Phage.Canada@gmail. com. https://talk.ictvonline.org/ictv/proposals/2018.081B.A.v1. Myxoctovirus.zip. Accessed 17 Apr 2019

123. Kropinski AM, Adriaenssens EM (2018) Create one new genus, Nickievirus, containing one new species, in the family Siphoviridae, order Caudovirales 2018.082B. Phage.Canada@gmail.com. https://talk.ictvonline.org/ictv/proposals/2018.082B.A.v1.Nicki evirus.zip. Accessed 17 Apr 2019

124. Kropinski AM, Adriaenssens EM (2018) Create one new genus, Otagovirus, containing two new species, in the family Myoviridae, order Caudovirales 2018.083B. Phage.Canada@gmail.com. https://talk.ictvonline.org/ictv/proposals/2018.083B.A.v1.Otago virus.zip. Accessed 17 Apr 2019

125. Kropinski AM, Adriaenssens EM (2018) Create one new genus, Peatvirus, containing one new species, in the family Myoviridae, order Caudovirales 2018.084B. Phage.Canada@gmail.com. https ://talk.ictvonline.org/ictv/proposals/2018.084B.A.v1.Peatvirus. zip. Accessed 17 Apr 2019

126. Magill D, Kropinski AM, Adriaenssens EM, Kulakov L (2018) Create one new genus, Plaisancevirus, containing one new species, in the family Myoviridae, order Caudovirales 2018.085B. Phage.Canada@gmail.com. https://talk.ictvonline.org/ictv/propo sals/2018.085B.A.v1.Plaisancevirus.zip. Accessed 17 Apr 2019

127. Kropinski AM, Adriaenssens EM, Colberg O, Carstens AB, Kot W, Hansen LH (2018) Create one new genus, Pollyceevirus, containing one new species, in the family Podoviridae, order Caudovirales 2018.086B. Phage.Canada@gmail.com. https:// talk.ictvonline.org/ictv/proposals/2018.086B.A.v1.Pollyceevi rus.zip. Accessed 17 Apr 2019

128. Kropinski AM, Adriaenssens EM (2018) Create one new genus, Rigallicvirus, containing one new species, in the family Siphoviridae, order Caudovirales 2018.087B.Phage.Canada@gmail. com. https://talk.ictvonline.org/ictv/proposals/2018.087B.A.v1. Rigallicvirus.zip. Accessed 17 Apr 2019

129. Kropinski AM, Adriaenssens EM (2018) Create one new genus, Ripduovirus, containing one new species, in the family Myoviridae, order Caudovirales 2018.088B. Phage.Canada@gmail.com. https://talk.ictvonline.org/ictv/proposals/2018.088B.A.v1.Ripdu ovirus.zip. Accessed 17 Apr 2019

130. Kropinski AM, Adriaenssens E (2018) Create one new genus, Risingsunvirus, containing one new species, in the family Myoviridae, order Caudovirales 2018.089B. Phage.Canada@gmail. com. https://talk.ictvonline.org/ictv/proposals/2018.089B.A.v1. Risingsunvirus.zip. Accessed 17 Apr 2019

131. Kropinski AM, Adriaenssens EM (2018) Create one new genus, Samunavirus, containing one new species, in the family Siphoviridae, order Caudovirales 2018.090B. Phage.Canada@gmail. com. https://talk.ictvonline.org/ictv/proposals/2018.090B.A.v1. Samunavirus.zip. Accessed 17 Apr 2019
132. Kropinski AM, Adriaenssens EM (2018) Create one new genus, Winklervirus, containing one new species, in the family Myoviridae, order Caudovirales 2018.091B. Phage.Canada@gmail.com. https://talk.ictvonline.org/ictv/proposals/2018.091B.A.v1.Winkl ervirus.zip. Accessed 17 Apr 2019

133. Kropinski AM, Adriaenssens EM (2018) Remove two species from the genus Xpl0virus, and reassign them to two new genera the family Siphoviridae, order Caudovirales 2018.092B. Phage. Canada@gmail.com. https://talk.ictvonline.org/ictv/propo sals/2018.092B.A.v2.Siphoviridae_2gen.zip. Accessed 17 Apr 2019

134. Krupovic M, Gillis A, le Marrec C (2018) Create one new genus, Gammatectivirus, containing one new species, in the family Tectiviridae 2018.093B. krupovic@ pasteur.fr; clehenaff@enscbp. fr. https://talk.ictvonline.org/ictv/proposals/2018.093B.A.v1. Gammatectivirus.zip. Accessed 17 Apr 2019

135. Knezevic P, Adriaenssens EM, Kropinski AM, Lavigne R (2018) Create two new species (unassigned genera) in the family Inoviridae 2018.094B. petar.knezevic@dbe.uns.ac.rs. https://talk. ictvonline.org/ictv/proposals/2018.094B.A.v1.Inoviridae_2sp. zip. Accessed 17 Apr 2019

136. Kropinski AM, Adriaenssens EM, Tong Y (2018) Create one new genus, Nanhaivirus, containing one new species, in the family Siphoviridae, order Caudovirales 2018.095B. Phage. Canada@gmail.com. https://talk.ictvonline.org/ictv/propo sals/2018.095B.A.v1.Nanhaivirus.zip. Accessed 17 Apr 2019

137. Kropinski AM, Adriaenssens EM, Lavigne R, Turner D, Yong Y (2018) Create one new genus, Napahaivirus, containing one new species, in the family Podoviridae, order Caudovirales 2018.096B. Phage.Canada@gmail.com. https://talk.ictvonline .org/ictv/proposals/2018.096B.A.v1.Napahaivirus.zip. Accessed 17 Apr 2019

138. Kropinski AM, Adriaenssens EM (2018) Create one new genus, Sanovirus, containing two new species, in the family Siphoviridae, order Caudovirales 2018.097B. Phage.Canada@gmail.com. https://talk.ictvonline.org/ictv/proposals/2018.097B.A.v1.Sanov irus.zip. Accessed 17 Apr 2019

139. Kropinski AM, Adriaenssens EM (2018) Create two new species in the genus Soupsvirus, in the family Siphoviridae, order Caudovirales 2018.098B. Phage.Canada@gmail.com. https:// talk.ictvonline.org/ictv/proposals/2018.098B.A.v2.Soupsvirus _2sp.zip. Accessed 17 Apr 2019

140. Kropinski AM, Adriaenssens EM Djurhuus AIMSM, Carstens AB, Neve H, Kot W, Hansen LH (2018) Create one new genus, Tabernariusvirus, containing one new species, in the family Podoviridae, order Caudovirales 2018.099B. Phage. Canada@gmail.com. https://talk.ictvonline.org/ictv/propo sals/2018.099B.A.v1.Tabernariusvirus.zip. Accessed 17 Apr 2019

141. Kropinski AM, Ackermann H-W, Adriaenssens E, Ehrlich K, Ehrlich M (2018) Create one new genus, Thornevirus, containing one new species, in the family Myoviridae, order Caudovirales 2018.100B.Phage.Canada@gmail.com. https://talk.ictvonline .org/ictv/proposals/2018.100B.A.v2.Thornevirus.zip. Accessed 17 Apr 2019

142. Kropinski AM, Adriaenssens EM (2018) Create one new genus, Tidunavirus, containing two new species, in the family Myoviridae, order Caudovirales 2018.101B. Phage.Canada@gmail.com. https://talk.ictvonline.org/ictv/proposals/2018.101B.A.v1.Tidun avirus.zip. Accessed 17 Apr 2019

143. Kropinski AM, Adriaenssens E (2018) Create one new genus, Trippvirus, containing one new species, in the family Siphoviridae, order Caudovirales 2018.102B. Phage.Canada@gmail.com. https://talk.ictvonline.org/ictv/proposals/2018.102B.A.v1.Tripp virus.zip. Accessed 17 Apr 2019 
144. Kropinski AM, Adriaenssens EM, Dias RS, Eller MR, de Paula SO (2018) Create one new genus, Vicosavirus, containing new two species, in the family Podoviridae, order Caudovirales 2018.103B. Phage.Canada@gmail.com. https://talk.ictvonline .org/ictv/proposals/2018.103B.A.v1.Vicosavirus.zip. Accessed 17 Apr 2019

145. Huang L, Wang H (2018) Create one new family, Ovaliviridae, containing one new genus, Alphaovalivirus, and one new species 2018.104B. huangl@sun.im.ac.cn. https://talk.ictvonline .org/ictv/proposals/2018.104B.A.v1.Ovaliviridae.zip. Accessed 17 Apr 2019

146. Bao H, Shahin K, Zhang H, Zhou Y, Pang M, Sun L, Schmidt S, Oliniran A, Wang R (2018) Create one new species, Salmonella virus SP116 in the genus Felixolvirus, subfamily Ounavirinae, family Myoviridae, order Caudovirales 2018.105B. ranwang@jaas.ac.cn. https://talk.ictvonline.org/ictv/propo sals/2018.105B.A.v2.Felixounavirus_sp.zip. Accessed 17 Apr 2019

147. Turner D, Adriaenssens EM, Kropinski AM (2018) Create eleven new species in the genus Friunavirus (renamed from Frilvirus in proposal 2018.007B), subfamily Autographivirinae, family Podoviridae, order Caudovirales 2018.106B. dann2.turner@uwe. ac.uk. https://talk.ictvonline.org/ictv/proposals/2018.106B.A.v1. Friunavirus_11sp.zip. Accessed 17 Apr 2019

148. Turner D, Adriaenssens EM, Kropinski AM (2018) Create one new genus, Lokivirus, containing two new species, in the family Siphoviridae, order Caudovirales 2018.107B. dann2. turner@uwe.ac.uk. https://talk.ictvonline.org/ictv/propo sals/2018.107B.A.v1.Lokivirus.zip. Accessed 17 Apr 2019

149. Turner D, Buttimer C, Coffey A, Neve H, Adriaenssens EM, Kropinski AM (2018) Create one new genus, Metrivirus, containing one new species, in the family Myoviridae, order Caudovirales 2018.108B.dann2.turner@uwe.ac.uk. https://talk.ictvonline.org/ ictv/proposals/2018.108B.A.v1.Metrivirus.zip. Accessed 17 Apr 2019

150. Turner D, Adriaenssens EM, Kropinski AM (2018) Create four new species in the genus Obolenskvirus, in the family Myoviridae, order Caudovirales 2018.109B. dann2.turner@uwe.ac.uk. https://talk.ictvonline.org/ictv/proposals/2018.109B.A.v1.Obole nskvirus_4sp.zip. Accessed 17 Apr 2019

151. Buttimer C, Turner D, Coffey A, Neve H, Kropinski AM, Adriaenssens E (2018) Create one new genus, Phimunavirus, containing four new species in the subfamily Autographivirinae, family Podoviridae, order Caudovirales 2018.110B. Phage. Canada@gmail.com.https://talk.ictvonline.org/ictv/propo sals/2018.110B.A.v1.Phimunavirus.zip. Accessed 17 Apr 2019

152. Turner D, Adriaenssens EM, Kropinski AM (2018) Create one new genus Vieuvirus, containing two new species, in the family Siphoviridae, order Caudovirales 2018.111B. dann2. turner@uwe.ac.uk. https://talk.ictvonline.org/ictv/propo sals/2018.111B.A.v1.Vieuvirus.zip. Accessed 17 Apr 2019

153. Kropinski AM, Adriaenssens EM, Peters DL, Dennis JJ (2018) Create 1 new genus, Delepquintavirus, containing one new species, in the family Siphoviridae, order Caudovirales 2018.112B. Phage.Canada@gmail.com. https://talk.ictvonline.org/ictv/propo sals/2018.112B.A.v1.Delepquintavirus.zip. Accessed $17 \mathrm{Apr}$ 2019

154. Kropinski AM, Adriaenssens EM, Tong Y (2018) Create one new genus, Efquatrovirus, containing fourteen new species, in the family Siphoviridae, order Caudovirales 2018.113B. Phage. Canada@gmail.com. https://talk.ictvonline.org/ictv/propo sals/2018.113B.A.v1.Efquatrovirus.zip. Accessed 17 Apr 2019

155. Kropinski AM, Adriaenssens EM, Laanto E, Sundberg L-R (2018) Create one new genus, Ficleduovirus, containing two new species, in the family Myoviridae, order Caudovirales
2018.114B. Phage.Canada@gmail.com. https://talk.ictvonline .org/ictv/proposals/2018.114B.A.v1.Ficleduovirus.zip. Accessed 17 Apr 2019

156. Kropinski AM, Adriaenssens EM, Bermúdez DEC (2018) Create one new genus, Fipvunavirus, containing two new species, in the family Podoviridae, order Caudovirales 2018.115B. Phage. Canada@gmail.com. https://talk.ictvonline.org/ictv/propo sals/2018.115B.A.v1.Fipvunavirus.zip. Accessed 17 Apr 2019

157. Kropinski AM, Adriaenssens EM (2018) Create one new genus, Jesfedecavirus, containing two new species, in the family Siphoviridae, order Caudovirales 2018.116B.Phage.Canada@gmail. com. https://talk.ictvonline.org/ictv/proposals/2018.116B.A.v1. Jesfedecavirus.zip. Accessed 17 Apr 2019

158. Yazdi M, Bouzari M, Ghaemi EA, Adriaenssens E, Kropinski A (2018) Create one new species (Escherichia virus Golestan) in the genus Klgvirus (rename Kagunavirus) in the subfamily Guernseyvirinae, family Siphoviridae, order Caudovirales 2018.117B.bouzari@sci.ui.ac.ir. https://talk.ictvonline.org/ictv/ proposals/2018.117B.A.v1.Kagunavirus_sp.zip. Accessed 17 Apr 2019

159. Barylski J, Enault F, Dutilh BE, Schuller MBP, Edwards RA, Gillis A, Klumpp J, Knezevic P, Krupovic M, Kuhn JH, Lavigne R, Oksanen HM, Sullivan MB, Jang H-B, Simmonds P, Aiewsakun P, Wittmann J, Tolstoy I, Brister JR, Kropinski AM, Adriaenssens EM (2018) Create one new family Herelleviridae in the order Caudovirales 2018.118B. evelien.adriaenssens@gmail. com. https://talk.ictvonline.org/ictv/proposals/2018.118B.A.v4. Herelleviridae.zip. Accessed 17 Apr 2019

160. Yazdi M, Bouzari M, Ghaemi EA, Adriaenssens E, Kropinski A (2018) Create one new genus, Gorganvirus, containing one new species, in the family Siphoviridae, order Caudovirales 2018.119B. bouzari@sci.ui.ac.ir. https://talk.ictvonline.org/ ictv/proposals/2018.119B.A.v1.Gorganvirus.zip. Accessed 17 Apr 2019

161. van Zyl L, Adriaenssens E, Kropinski A (2018) Create one new genus, Magadivirus, containing one new species, in the family Siphoviridae, order Caudovirales 2018.120B. vanzyllj@gmail. com. https://talk.ictvonline.org/ictv/proposals/2018.120B.A.v1. Magadivirus.zip. Accessed 17 Apr 2019

162. van Zyl L, Adriaenssens E, Kropinski A (2018) Create one new genus, Shalavirus, containing one new species, in the family Myoviridae, order Caudovirales 2018.121B. vanzyllj@gmail. com. https://talk.ictvonline.org/ictv/proposals/2018.121B.A.v1. Shalavirus.zip. Accessed 17 Apr 2019

163. Adriaenssens EM, Kropinski AM (2018) Create one new genus, Sourvirus, containing one new species, in the family Siphoviridae, order Caudovirales 2018.122B. Phage.Canada@gmail.com. https://talk.ictvonline.org/ictv/proposals/2018.122B.A.v1.Sourv irus.zip. Accessed 17 Apr 2019

164. Leigh BA, Breitbart M, Bamford DH, Dishaw LJ, Adriaenssens E, Kropinski A, Oksanen HM (2018) Create one new species, Pseudoalteromonas virus Cr39582, in the genus Corticovirus, family Corticoviridae 2018.123B. hanna.oksanen@ @elsinki.fi. https://talk.ictvonline.org/ictv/proposals/2018.123B.A.v1.Corti covirus_sp.zip. Accessed 17 Apr 2019

165. van Zyl L, Adriaenssens E, Kropinski A (2018) Create one new genus, Vhulanivirus, containing one new species, in the family Siphoviridae, order Caudovirales 2018.124B. vanzyllj@gmail. com. https://talk.ictvonline.org/ictv/proposals/2018.124B.A.v1. Vhulanivirus.zip. Accessed 17 Apr 2019

166. Kropinski AM, Tolstoy I, Adriaenssens E (2018) Create sixtyone new species in existing genera of the family Myoviridae 2018.125B. evelien.adriaenssens@gmail.com. https://talk.ictvo nline.org/ictv/proposals/2018.125B.A.v1.Myoviridae_61sp.zip. Accessed 17 Apr 2019 
167. Kropinski AM, Adriaenssens EM (2018) Create one new genus, Myunavirus, containing one new species, in the family Siphoviridae, order Caudovirales 2018.126B. Phage.Canada@gmail. com. https://talk.ictvonline.org/ictv/proposals/2018.126B.A.v1. Myunavirus.zip. Accessed 17 Apr 2019

168. Kropinski AM, Adriaenssens EM (2018) Create one new genus, Novosibvirus, containing one new species, in the family Siphoviridae, order Caudovirales 2018.127B. Phage.Canada@gmail. com. https://talk.ictvonline.org/ictv/proposals/2018.127B.A.v1. Novosibvirus.zip. Accessed 17 Apr 2019

169. Kropinski AM, Adriaenssens EM, Azeredo J, Oliveira H (2018) Create one new genus, Priunavirus, containing one new species, in the family Siphoviridae, order Caudovirales 2018.128B. Phage.Canada@gmail.com. https://talk.ictvonline.org/ictv/propo sals/2018.128B.A.v1.Priunasvirus.zip. Accessed 17 Apr 2019

170. Kropinski AM, Adriaenssens EM (2018) Create one new genus, Sugarlandvirus, containing two new species, in the family Siphoviridae, order Caudovirales 2018.129B. Phage.Canada@gmail. com. https://talk.ictvonline.org/ictv/proposals/2018.129B.A.v1. Sugarlandvirus.zip. Accessed 17 Apr 2019

171. Kropinski AM, Adriaenssens EM (2018) Create three new genera, each containing one new species, in the subfamily Tunavirinae, in the family Siphoviridae, order Caudovirales 2018.130B. Phage.Canada@gmail.com. https://talk.ictvonline.org/ictv/propo sals/2018.130B.A.v1.Tunavirinae_3gen.zip. Accessed 17 Apr 2019

172. Kropinski AM, Adriaenssens EM, Bermúdez DEC (2018) Create one new genus, Unahavirus, containing four new species, in the family Siphoviridae, order Caudovirales 2018.131B. Phage. Canada@gmail.com. https://talk.ictvonline.org/ictv/propo sals/2018.131B.A.v1.Unahavirus.zip. Accessed 17 Apr 2019

173. Adriaenssens E, Tolstoy I, Kropinski A (2018) Rename one species and create two new species within the order Caudovirales 2018.132B. evelien.adriaenssens@gmail.com. https://talk.ictvo nline.org/ictv/proposals/2018.132B.A.v1.Caudovirales_2sp1r en.zip. Accessed 17 Apr 2019

174. Adriaenssens EM, Kropinski AM (2018) Create one new genus, Avunavirus, containing five new species in the subfamily Vequintavirinae, in the family Myoviridae, order Caudovirales 2018.133B. Phage.Canada@gmail.com. https://talk.ictvonline .org/ictv/proposals/2018.133B.A.v1.Vequintavirinae_1gen5 sp.zip. Accessed 17 Apr 2019

175. Kropinski AM, Adriaenssens EM (2018) Create one new genus, Cetovirus, containing three new species, in the family Siphoviridae, order Caudovirales. 2018.134B. Phage. Canada@gmail.com. https://talk.ictvonline.org/ictv/propo sals/2018.134B.A.v1.Cetovirus.zip. Accessed 17 Apr 2019

176. Shahin K, Bouzari M, Wang R (2018) Create one new species, Shigella virus ISF002, within the genus T1virus (rename Tunavirus), subfamily Tunavirinae, in the family Siphoviridae, order Caudovirales 2018.135B. bouzari@sci.ui.ac.ir; ranwang@jaas.ac.cn. https://talk.ictvonline.org/ictv/propo sals/2018.135B.A.v1.Tunavirus_sp.zip. Accessed 17 Apr 2019

177. Adriaenssens EM, Kropinski AM (2018) Move species Mycobacterium virus TA17a to the genus Rosebushvirus, subfamily Bclasvirinae, in the family Siphoviridae, order Caudovirales 2018.136B. Phage.Canada@gmail.com.https://talk.ictvonline .org/ictv/proposals/2018.136B.A.v1.Siphoviridae_spmov.zip. Accessed 17 Apr 2019

178. Adriaenssens EM, Kropinski AM (2018) Rename five species and three genera in the family Siphoviridae, in the family Siphoviridae, order Caudovirales 2018.137B. Phage. Canada@gmail.com. https://talk.ictvonline.org/ictv/propo sals/2018.137B.A.v1.Siphoviridae_ren5sp.zip. Accessed 17 Apr 2019
179. Adriaenssens EM, Kropinski AM (2018) Delete one species from the genus Tulanevirus, in the family Myoviridae, order Caudovirales 2018.138B.Phage.Canada@gmail.com. https://talk.ictvo nline.org/ictv/proposals/2018.138B.A.v1.Tulanevirus_sprem.zip. Accessed 17 Apr 2019

180. Kropinski AM, Adriaenssens EM, Toussaint A, Pourcel C (2018) Create one new genus (Beetrevirus) including four new species, in the family Siphoviridae, order Caudovirales 2018.140B. Phage.Canada@gmail.com. https://talk.ictvonline.org/ictv/propo sals/2018.140B.A.v1.Beetrevirus.zip. Accessed 17 Apr 2019

181. Ghabrial S, Nibert M (2018) Rename one species Helminthosporium victoriae $145 \mathrm{~S}$ virus (genus Chrysovirus) to Helminthosporium victoriae virus $145 \mathrm{~S}$ in the family Chrysoviridae 2018.001F. mnibert@hms.harvard.edu. https://talk.ictvo nline.org/ictv/proposals/2018.001F.A.v2.Chrysovirus_spren.zip. Accessed 17 Apr 2019

182. Lang AS, Vlok M, Suttle CA (2018) Assign four new and two unassigned genera to the family Marnaviridae in the order Picornavirales 2018.002F. aslang@mun.ca. https://talk.ictvo nline.org/ictv/proposals/2018.002F.A.v2.Marnaviridae_4gen. zip. Accessed 17 Apr 2019

183. Ayllón MA, Donaire L, Marzano S-Y, Petrzik K, Sánchez-Gracia A, Rozas J, Turina M (2018) Establish one new family, Botourmiaviridae, including one existing (Ourmiavirus) and three new genera (Botoulivirus, Magoulivirus and Scleroulivirus) 2018.003F. mariaangeles.ayllon@upm.es. https://talk.ictvonline .org/ictv/proposals/2018.003F.A.Botourmiaviridae.zip. Accessed 17 Apr 2019

184. Kotta-Loizou I, Ghabrial SA, Castón JR, Coutts RHA, Hillman BI, Jiang D, Kim D-H, Moriyama H, Suzuki N (2018) Rename one genus, adding one new genus and sixteen new species in the family Chrysoviridae 2018.004F. i.kotta-loizou13@imperial. ac.uk. https://talk.ictvonline.org/ictv/proposals/2018.004F.A.v3. Betachrysovirus.zip. Accessed 17 Apr 2019

185. Scheets K, Surapathrudu K, Miller WA (2018) Create three new subfamilies in family Tombusviridae: Procedovirinae, Regressovirinae and Calvusvirinae 2018.001P. kay.scheets@okstate.edu. https://talk.ictvonline.org/ictv/proposals/2018.001P.A.v1.Tombu sviridae 3subfam.zip. Accessed 17 Apr 2019

186. Melzer MJ, Freitas-Astúa J, Rodriguez JCV, Roy A, Wei G (2018) Create one new family Kitaviridae comprising three previously unassigned genera, Cilevirus, Blunervirus and Higrevirus 2018.002P. melzer@hawaii.edu. https://talk.ictvonline.org/ictv/ proposals/2018.002P.A.Kitaviridae.zip. Accessed 17 Apr 2019

187. Scheets K (2018) Create one new species in genus Pelarspovirus in the family Tombusviridae 2018.003P. kay.scheets@okstate. edu. https://talk.ictvonline.org/ictv/proposals/2018.003P.A.v1. Pelarspovirus_sp.zip. Accessed 17 Apr 2019

188. Scheets K (2018) Correct one species name (Cucumber Bulgarian latent virus) in the family Tombusviridae 2018.004P. kay.scheets@okstate.edu. https://talk.ictvonline.org/ictv/propo sals/2018.004P.A.v1.Tombusvirus_spren.zip. Accessed 17 Apr 2019

189. Marais A, Candresse T (2018) Create one new species (Mume virus $A$ ) in the genus Capillovirus, family Betaflexiviridae, order Tymovirales 2018.005P. armelle.marais-colombel@inra.fr. https ://talk.ictvonline.org/ictv/proposals/2018.005P.A.v1.Capillovir us_spa.zip. Accessed 17 Apr 2019

190. Petrzik K, Pribylova J, Koloniuk I, Spak J (2018) Create one new species (Currant virus A) in the genus Capillovirus, family Betaflexiviridae, order Tymovirales 2018.006P. petrzik@umbr. cas.cz. https://talk.ictvonline.org/ictv/proposals/2018.006P.A.v1. Capillovirus_spb.zip. Accessed 17 Apr 2019

191. Jo Y, Song M-K, Choi H, Park J-S, Lee J-W, Lian S, Lee BC, Cho WK (2018) Create one new species (Grapevine virus T) in the genus Foveavirus, family Betaflexiviridae, order Tymovirales 
2018.007P. wonkyong@gmail.com. https://talk.ictvonline.org/ ictv/proposals/2018.007P.A.v1.Foveavirus_sp.zip. Accessed 17 Apr 2019

192. Veerakone S, Liefting LW, Tang J, Ward LI (2018) Create one new species (Actinidia seed borne latent virus) in the genus Prunevirus, family Betaflexiviridae, order Tymovirales 2018.008P. stella.veerakone@mpi.govt.nz. https://talk.ictvonline.org/ictv/ proposals/2018.008P.A.v1.Prunevirus_sp.zip. Accessed 17 Apr 2019

193. Xin M, Wang X (2018) Create one new genus, Wamavirus, containing one new species, family Betaflexiviridae, order Tymovirales 2018.009P.xfwang@ippcaas.cn. https://talk.ictvonline.org/ ictv/proposals/2018.009P.A.v1.Wamavirus.zip. Accessed 17 Apr 2019

194. Hassan M, Tzanetakis IE (2018) Create one new species (Blackberry virus $A$ ) in the genus Vitivirus, family Betaflexiviridae, order Tymovirales 2018.010P. itzaneta@uark.edu. https://talk. ictvonline.org/ictv/proposals/2018.010P.A.v1.Vitivirus_spa.zip. Accessed 17 Apr 2019

195. Blouin AG, Chooi KM, Warren B, Napier KR, Barrero RA, MacDiarmid RM (2018) Create one new species (Grapevine virus $G)$ in the genus Vitivirus, family Betaflexiviridae, order Tymovirales 2018.011P. Arnaud.blouin@plantandfood.co.nz. https:// talk.ictvonline.org/ictv/proposals/2018.011P.A.v1.Vitivirus_spb. zip. Accessed 17 Apr 2019

196. Candresse T, Marais A (2018) Create one new species (Grapevine virus $H$ ) in the genus Vitivirus, family Betaflexiviridae, order Tymovirales 2018.012P. thierry.candresse@inra.fr. https://talk. ictvonline.org/ictv/proposals/2018.012P.A.v1.Vitivirus_spc.zip. Accessed 17 Apr 2019

197. Blouin AG, Chooi KM, Warren B, Napier KR, Barrero RA, MacDiarmid RM (2018) Create one new species (Grapevine virus I) in the genus Vitivirus, family Betaflexiviridae, order Tymovirales 2018.013P. Arnaud.blouin@ @lantandfood.co.nz. https:// talk.ictvonline.org/ictv/proposals/2018.013P.A.v1.Vitivirus_spd. zip. Accessed 17 Apr 2019

198. Diaz-Lara A, Golino D, Al Rwahnih M (2018) Create one new species (Grapevine virus $J$ ) in the genus Vitivirus, family Betaflexiviridae, order Tymovirales 2018.014P. malrwahnih@ucdavis. edu. https://talk.ictvonline.org/ictv/proposals/2018.014P.A.v1. Vitivirus_spe.zip. Accessed 17 Apr 2019

199. Silva JMF, Al Rwahnih M, Blawid R, Nagata T, Fajardo TVM (2018) Create one new species (Grapevine enamovirus 1) in the genus Enamovirus, family Luteoviridae 2018.015P. thor. fajardo@embrapa.br. https://talk.ictvonline.org/ictv/propo sals/2018.015P.A.v1.Enamovirus_spa.zip. Accessed 17 Apr 2019

200. Kanakala S, Miller WA (2018) Create one new species (Citrus vein enation virus) in the genus Enamovirus, family Luteoviridae 2018.016P.wamiller@iastate.edu. https://talk.ictvo nline.org/ictv/proposals/2018.016P.A.v1.Enamovirus_spb. zip. Accessed 17 Apr 2019

201. Kanakala S, Carino EJ, Miller WA (2018) Create one new species (Nectarine stem pitting associated virus) in the genus Luteovirus, family Luteoviridae 2018.017P. wamiller@iastate. edu. https://talk.ictvonline.org/ictv/proposals/2018.017P.A.v1. Luteovirus_spa.zip. Accessed 17 Apr 2019

202. Kanakala S, Olawole OI, Miller WA (2018) Create one new species (Cherry associated luteovirus) in the genus Luteovirus, family Luteoviridae 2018.018P.wamiller@iastate.edu. https:// talk.ictvonline.org/ictv/proposals/2018.018P.A.v1.Luteovirus _spb.zip. Accessed 17 Apr 2019

203. Navas-Castillo J, Fiallo-Olivé E, Zerbini FM (2018) Rename one species Pepper vein yellows virus and create five new species in the genus Polerovirus, family Luteoviridae 2018.019P. jnavas@eelm.csic.es. https://talk.ictvonline.org/ictv/propo
sals/2018.019P.A.v1.Polerovirus_5sp1ren.zip. Accessed 17 Apr 2019

204. Di Serio F, Navarro B, Minutolo M, Alioto D (2018) Create one new species (Citrus coguvirus) in one new genus (Coguvirus) in the order Bunyavirales 2018.020P. francesco. diserio@ipsp.cnr.it. https://talk.ictvonline.org/ictv/propo sals/2018.020P.A.v1.Coguvirus.zip. Accessed 17 Apr 2019

205. Navarro B, Di Serio F, Aboughanem-Sabanadzovic N (2018) Create one new species (Privet idaeovirus) in the genus Idaeovirus 2018.021P. beatriz.navarro@ipsp.cnr.it. https://talk.ictvo nline.org/ictv/proposals/2018.021P.A.v1.Idaeovirus_sp.zip. Accessed 17 Apr 2019

206. Scheets K (2018) Create one new species (Potato necrosis virus) in genus Alphanecrovirus in the family Tombusviridae 2018.022P. kay.scheets@okstate.edu. https://talk.ictvonline .org/ictv/proposals/2018.022P.A.v1.Alphanecrovirus_sp.zip. Accessed 17 Apr 2019

207. Teycheney P-Y, Muller E, Umber M, Geering A (2018) Create eleven new species and rename one species in the genus Badnavirus, and create two new species in the genus Caulimovirus, family Caulimoviridae, order Ortervirales 2018.023P. teycheney@cirad.fr. https://talk.ictvonline.org/ictv/propo sals/2018.023P.A.v1.Caulimoviridae_13sp.zip. Accessed 17 Apr 2019

208. Fuchs M, Bar-Joseph M, Candresse T, Martelli GP, Maree H, Melzer MJ, Menzel W, Minafra A, Sabanadzovic S (2018) Create two new species in the family Closteroviridae 2018.024P. mf13@cornell.edu. https://talk.ictvonline.org/ictv/propo sals/2018.024P.A.v1.Closteroviridae_2sp.zip. Accessed 17 Apr 2019

209. Adkins S, Naidu R, Resende R, Turina M, Whitfield A, Lambert A, Zerbini FM (2018) Create seven new species in the genus Orthotospovirus, family Tospoviridae, order Bunyavirales 2018.025P. scott.adkins@ ars.usda.gov. https://talk.ictvo nline.org/ictv/proposals/2018.025P.A.v1.Orthotospovirus_7sp. zip. Accessed 17 Apr 2019

210. Wylie S, Kreuze JF, Lopez-Moya JJ, Makinen K, InoueNagata AK, Ohshima K, Wang A (2018) Create seven new species in genus Potyvirus, family Potyviridae 2018.026P. s.wylie@murdoch.edu.au.https://talk.ictvonline.org/ictv/propo sals/2018.026P.A.v1.Potyvirus_7sp.zip. Accessed 17 Apr 2019

211. Wylie S, Kreuze JF, Lopez-Moya JJ, Makinen K, Inoue-Nagata AK, Ohshima K, Wang A (2018) Create two unassigned species in family Potyviridae 2018.027P. s.wylie@murdoch.edu. au. https://talk.ictvonline.org/ictv/proposals/2018.027P.A.v1. Potyviridae_2sp.zip. Accessed 17 Apr 2019

212. Varsani A, Roumagnac P, Lett J-M, Martin DP (2018) Create three new species in the genus Mastrevirus, one new species in the genus Becurtovirus, two new species in the genus Grablovirus, family Geminiviridae 2018.028P. Arvind.varsani@asu.edu. https://talk.ictvonline.org/ictv/proposals/2018.028P.A.v1.Gemin iviridae_6sp.zip. Accessed 17 Apr 2019

213. Fiallo-Olive E, Navas-Castillo J, Zerbini FM (2018) Create twenty-eight species and abolish five species in the genus Begomovirus, family Geminiviridae 2018.029P. efiallo@eelm.csic.es. https://talk.ictvonline.org/ictv/proposals/2018.029P.A.v2.Begom ovirus_28sp_5sprem.zip. Accessed 17 Apr 2019

214. Varsani A, Zerbini FM (2018) Rename two species in the family Alphasatellitidae 2018.030P. Arvind.varsani@asu.edu. https:// talk.ictvonline.org/ictv/proposals/2018.030P.A.v1.Alphasatellitid ae_2spren.zip. Accessed 17 Apr 2019

215. Sabanadzovic S, Aboughanem-Sabanadzovic N, Dolja VV, Krupovic M, Koonin EV, Valverde RA (2018) Recognize the family Endornaviridae as a taxon of positive-sense RNA viruses in the ICTV framework 2018.031P. ss501@ msstate.edu. https 
://talk.ictvonline.org/ictv/proposals/2018.031P.A.v2.Endornavir idae_reclassif.docx. Accessed 17 Apr 2019

216. Sabanadzovic S, Nibert ML, Krupovic M, Tzanetakis IE, Valverde RA (2018) Create five new species in the genus Amalgavirus and create one new genus, Zybavirus, containing one new species, all in the family Amalgaviridae 2018.032P. ss501@ msstate.edu. https://talk.ictvonline.org/ictv/propo sals/2018.032P.A.v1.Amalgaviridae_rev.zip. Accessed 17 Apr 2019

217. Mushegian AR, Davison AJ (2019) Virology division news: Michael Adams: new Life Member of the International Committee on Taxonomy of Viruses (ICTV). Arch Virol. https://doi. org/10.1007/s00705-019-04290-1

218. Wolf YI, Kazlauskas D, Iranzo J, Lucía-Sanz A, Kuhn JH, Krupovic M, Dolja VV, Koonin EV (2018) Origins and evolution of the global RNA virome. MBio 9:e02329-18. https://doi. org/10.1128/mBio.02329-18
219. Kuhn JH, Wolf YI, Krupovic M, Zhang YZ, Maes P, Dolja VV, Koonin EV (2019) Classify viruses - the gain is worth the pain. Nature 566:318-320. https://doi.org/10.1038/d41586-019-00599 $-8$

220. Wolf YI, Kazlauskas D, Iranzo J, Lucía-Sanz A, Kuhn JH, Krupovic M, Dolja VV, Koonin EV (2019) Reply to Holmes and Duchêne, "Can sequence phylogenies safely infer the origin of the global virome?": deep phylogenetic analysis of RNA viruses is highly challenging but not meaningless. MBio 10:e0542-19. https://doi.org/10.1128/mBio.00542-19

Publisher's Note Springer Nature remains neutral with regard to jurisdictional claims in published maps and institutional affiliations.

\section{Affiliations}

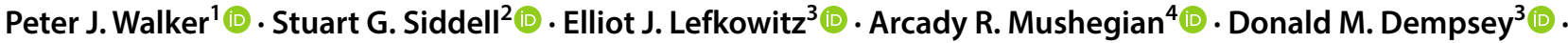

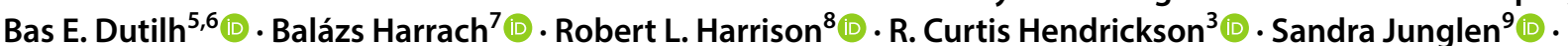

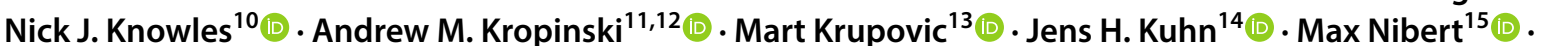

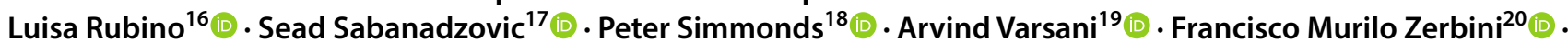
Andrew J. Davison ${ }^{21}$ (1)

Peter J. Walker

peter.walker@uq.edu.au

Stuart G. Siddell

stuart.siddell@bristol.ac.uk

Elliot J. Lefkowitz

elliot1@uab.edu

Donald M. Dempsey

ddempsey@uab.edu

Bas E. Dutilh

bedutilh@gmail.com

Balázs Harrach

harrach.balazs@agrar.mta.hu

Robert L. Harrison

robert.1.harrison@ars.usda.gov

R. Curtis Hendrickson

curtish@uab.edu

Sandra Junglen

sandra.junglen@charite.de

Nick J. Knowles

nick.knowles@ pirbright.ac.uk

Andrew M. Kropinski

phage.canada@gmail.com

Mart Krupovic

mart.krupovic@pasteur.fr

Jens H. Kuhn

kuhnjens@mail.nih.gov

Max Nibert

mnibert@hms.harvard.edu

Luisa Rubino

luisa.rubino@cnr.it
Sead Sabanadzovic

ssabanadzovic@entomology.msstate.edu

Peter Simmonds

peter.simmonds@ndm.ox.ac.uk

Arvind Varsani

arvind.varsani@asu.edu

Francisco Murilo Zerbini

zerbini@ufv.br

Andrew J. Davison

andrew.davison@glasgow.ac.uk

1 School of Biological Sciences, The University of Queensland, Saint Lucia, QLD 4072, Australia

2 School of Cellular and Molecular Medicine, Faculty of Life Sciences, University of Bristol, University Walk, Bristol BS8 1TD, UK

3 Department of Microbiology, University of Alabama at Birmingham (UAB), BBRB 276, 845 19th ST South, Birmingham, AL 35294-2170, USA

4 Division of Molecular and Cellular Biosciences, National Science Foundation, 2415 Eisenhower Avenue, Alexandria, VA 22314, USA

5 Theoretical Biology and Bioinformatics, Department of Biology, Utrecht University, Padualaan 8, Room Z-509, 3584 CH Utrecht, The Netherlands

6 Centre for Molecular and Biomolecular Informatics, Radboud University Medical Center (Radboudumc), Geert Grooteplein 26, 6525 GA Nijmegen, The Netherlands

7 Centre for Agricultural Research, Institute for Veterinary Medical Research, Hungarian Academy of Sciences, Hungária krt. 21, Budapest 1143, Hungary 
8 Invasive Insect Biocontrol and Behavior Laboratory, USDA-ARS, 10300 Baltimore Avenue, Bldg 007, BARC-West, Beltsville, MD 20705, USA

9 Institute of Virology, Charité-Universitätsmedizin, corporate member of Free University Berlin,

Humboldt-University Berlin, Berlin Institute of Health, Berlin, Germany

10 The Pirbright Institute, Ash Road, Pirbright, Surrey GU24 0NF, UK

11 Department of Food Science, University of Guelph, Guelph, Ontario N1G 2W1, Canada

12 Department of Pathobiology, University of Guelph, Guelph, Ontario N1G 2W1, Canada

13 Department of Microbiology, Institut Pasteur, 25 rue du Dr Roux, 75015 Paris, France

14 NIH/NIAID/DCR Integrated Research Facility at Fort Detrick (IRF-Frederick), B-8200 Research Plaza, Fort Detrick, Frederick, MD 21702, USA

15 Department of Microbiology and Immunobiology, Harvard Medical School, 77 Ave Louis Pasteur, Boston, MA 02115, USA
16 Istituto per la Protezione Sostenibile delle Piante, CNR, UOS Bari, Via Amendola 165/A, 70126 Bari, Italy

17 Department of Biochemistry, Molecular Biology, Entomology and Plant Pathology, Mississippi State University, 100 Old Hwy 12 Mail Stop 9775, Mississippi State, MS 39762, USA

18 Nuffield Department of Experimental Medicine, University of Oxford, Peter Medawar Building, South Parks Road, Oxford OX1 3PS, UK

19 The Biodesign Center for Fundamental and Applied Microbiomics, School of Life Sciences, Center for Evolution and Medicine, Arizona State University, P.O. Box 874701, Tempe, AZ 85287-4701, USA

20 Departamento de Fitopatologia/BIOAGRO, Universidade Federal de Viçosa, Viçosa, MG 36570-900, Brazil

21 MRC-University of Glasgow Centre for Virus Research, Sir Michael Stoker Building, 464 Bearsden Road, Glasgow G61 1QH, UK 